$B$ e $m$ erkunge $n$.

Zur Herleitung der mittleren Oerter der Vergl. *e wurden von Juli 9 bis Juli 19 der grossen Annäherung an den $\mathrm{Pol}$ wegen die strengen Formeln angewandt und boi den scheinbaren Oertern die von $2 \&$ abhängigen Glieder und der nicht unbedeutende Betrag der tägliçhen Aberration berücksichtigt. - Fine neuerdings vorgenommene Untersuchung der Mikrometerschraube hat einen merklichen fortschreitenden Gang derselben ergeben. Bei der Reduction der Schraubenablesungen ist dieser Gang jetzt in Rechnung gezogen worden; bei einzelnen schon früber publicirten Beobachtungen dieses Cometen ist dies nicht der Fall gewesen und erklären sich daraus die kleinen Abweichungen in $f$ zwischen den vorstehenden und den früher gegebenen Werthen. - Die Beobachtungen werden meist bei 144 facher Vergrösserung angestellt, einige Male sogar bei 192facher.

In Betreff der Gestalt des Cometen und der Witterungsverhältnisse ist Folgendes zu bemerken:

Juni 23. \&4 scheint Kern gleich $* 12^{\mathrm{m}}$ zu haben.

30. \&4 hat Verdichtung und erscheint gekörnt, Schweif nicht bemerkbar, Luftzustand II-III. \& schein.
Juli 4. \&4 erscheint wie ein ausserordentliel schwacher Nebel mit etwas Verdichtung, II, $\mathbb{C}$.

9. \&f wie * $11^{\mathrm{m}}$ trotz $\mathbb{C}$ und Cirri.

14. Ziemlich hell, mit etwas Verdichtung, II.

18. Planetarische Verdichtung von $15^{\prime \prime}$ diam. und der Helligkeit eines * 10m5; Durchmesser des ganzen \& 1 .

19. Deutlicher punktartiger Kern, um diesen planetarische Verdichtung von etwa 0'2 Durchmesser und um diese noch eine Hülle von '2' (o Durchmesser.

20. Durchmesser 2', Verdichtung, II.

22. \& mit deutlichem punktartigen Kern, Luftzustand I-III.

Aug. 12. $\&$ erscheint als verwaschoner, in der Mitte etwas verdichteter Nebel von 0'7 Durch. messer, so hell als $* 12^{\mathrm{m}}-13^{\mathrm{m}}$, II.

14. \& gleicht einem schwachen, von $O$ nach W vorlängerten Nebel, etwas $\nabla$ erdichtung, II.

17. 1'5 Durchmesser, II.

\title{
Ueber einen neuen Nebel im Sternbilde des Schwans.
}

Durch Lord Lindsay (Dunecht Observatory) erhielt ich vor einigen Tagen die Nachricht, dass Mr. T. W. Webb am 14. November 1879 einen kleinen Nebel oder Nebelstern im Schwan entdeckt habe.

Das Object, welches ich gestern, am 29. Novbr., beobachten konnte, als sich der Himmel in später Abendstunde auf einige Zoit aufhellte, ist identisch mit einem Stern $8 \mathrm{~m} 5$ der Bonner Durchmusterung $\left(-+41^{\circ} \mathrm{Nr}\right.$. 4004). Mit stärkerer Vergrösserung erschien dieser Stern als ein runder Nebel mit verwaschenem Rande von etwa 3" bis 4" Durchmesser. Das Spectrum schien anf den ersten Blick aus einer einzigen Linie zu bestehen, ein ganz schwaches continuirliches Spectrum wurde bei weiterer Beobachtung zu beiden Seiten dieser hellen Linie vermuthet. Der helle Mondschein und dor mit leichtem Schleier überzogene Himmelsgrund erschwerten die Beobachtungen sebr. Eine Positionsbestimmung der hellen Linie im Spectrum war nicht möglich, da der Himmel sich wieder stärker bewölkte.

Potsdam, Nov. 30, 1879.

\section{Berichtigung.}

Die in Nr. 2284 Seite 62 dieses Blattes mitgetheilte zweite Beobachtung des Cometen II 1867 ist, wie es auch in der Uebersehrift angegeben, vom 8 . Juli und nicht rom 7. Juli.

\section{In halt:}

Za Nr. 2289. Breusing. Nonius oder Vernier? 129. - A. Marth. Data for computing the positions of the Satellites of Mars 1879 . I33. -Meissel. Beitrag zur Sphärik. 139. - C. Bruhns. Beobachtungen an I2füssigen Aequatoreal der Leipziger Sternwarte. 14 I. H. C. V'ogel. Leber einen neuen Nebel im Sternbilde des Schwans. 143. - Berichtigung. 143. 